\title{
Aspectos epidemiológicos da vassoura-de-bruxa do cupuaçuzeiro na microrregião de Belém, $\mathbf{P A}^{(1)}$
}

\author{
Ruth Linda Benchimol(2), Fernando Carneiro de Albuquerque ${ }^{(2)}$ e Risaldo Muniz do Nascimento ${ }^{(3)}$
}

\begin{abstract}
Resumo - Foi conduzido experimento no campo da Embrapa-Centro de Pesquisa Agropecuária do Trópico Úmido, em Belém, PA ( $\left.1^{\circ} 28^{\prime} \mathrm{S} ; 4^{\circ} 27^{\prime} \mathrm{W}\right)$, de junho/91 a maio/94, com plantas de cupuaçuzeiro (Theobroma grandiflorum (Willd. ex Spreng.) K. Schum.) com sete anos de idade, objetivando acompanhar o desenvolvimento de vassouras-de-bruxa vegetativas causadas por Crinipellis perniciosa ((Stahel) Singer), desde a sua emissão até a produção de basidiocarpos. Foram determinados coeficientes de correlação entre as variáveis epidemiológicas (emissão de vassouras, período verde, período de secamento, período pré-frutificativo, produção de basidiocarpos) e as variáveis climáticas (umidade relativa do ar máxima, brilho solar, precipitação pluvial). A emissão de vassouras vegetativas foi maior em julho e agosto. Estas permaneceram verdes por 34,9 a 65,2 dias e secaram entre 5,8 e 10 dias. Após sua emissão, as vassouras demoraram de 79,5 a 347 dias para iniciar a produção de basidiocarpos, que ocorreu principalmente a partir de maio, com pico em junho. As variáveis climáticas, umidade relativa do ar máxima e brilho solar, correlacionaram-se significativamente apenas com a produção de basidiocarpos, com efeito negativo e positivo, respectivamente.
\end{abstract}

Termos para indexação: Crinipellis perniciosa, Theobroma grandiflorum, chuva, precipitação, umidade relativa, brilho solar.

\section{Epidemiological aspects of witches' broom disease of cupuassu tree in the microregion of Belém, PA, Brazil}

\begin{abstract}
A field experiment was set at Embrapa-Centro de Pesquisa Agropecuária do Trópico Úmido, in Belém, PA $\left(1^{\circ} 28^{\prime} \mathrm{S} ; 48^{\circ} 27^{\prime} \mathrm{W}\right)$, from June/91 to May/94, aiming to follow vegetative brooms caused by Crinipellis perniciosa ((Stahel) Singer), produced in seven-year-old cupuassu trees (Theobroma grandiflorum (Willd. ex Spreng.) K. Schum.), from emission to basidiocarp production. Correlation coefficients between epidemiological variables (number of vegetative brooms, green and drying period, latent period, basidiocarp production) and climatic variables (maximum relative humidity of the air, sunshine, rainfall) were determined. The emission of vegetative brooms was observed mostly in July and August. Brooms remained green for 34.9 to 65.2 days and dried after 5.8 to 10 days. After emission, vegetative brooms delayed from 79.5 to 347 days to begin basidiocarp production, which occurred mostly from May on, with the peak in June. The correlations between the climatic variables, maximum relative humidity of air and sunshine were significant only for basidiocarp production, with negative and positive effects, respectively.
\end{abstract}

Index terms: Crinipellis perniciosa, Theobroma grandiflorum, rain, precipitation, relative humidity, sunshine.

\footnotetext{
${ }^{(1)}$ Aceito para publicação em 14 de abril de 2000.

Trabalho desenvolvido com apoio do Convênio Embrapa-Centro de Pesquisa Agropecuária do Trópico Úmido (CPATU)/ JICA.

(2) Embrapa-CPATU, Caixa Postal 48, CEP 66017-970 Belém, PA. E-mail: rlinda@cpatu.embrapa.br, carneiro@cpatu.embrapa.br

(3) CODENPA, Rodovia PA 140, km 14, CEP 66000-000 Belém, PA.
}

\section{Introdução}

A vassoura-de-bruxa (Crinipellis perniciosa (Stahel) Singer) é a doença que mais ameaça os plantios de cupuaçuzeiro (Theobroma grandiflorum (Willd. ex Spreng.) K. Schum.) na Região Amazônica. Embora os danos econômicos acarretados por essa doença em plantios de cupuaçuzeiro ainda não te- 
nham sido quantificados de forma precisa, sabe-se que os ramos vegetativos, almofadas florais e frutos atacados pela doença secam e morrem, pois interferem de forma significativa na área fotossintética e na produtividade da planta (Nunes et al., 1996; Stein et al., 1996, 1997; Alves et al., 1997).

Apesar de bem estudada no cacaueiro (Theobroma cacao L.) (Hernandez, 1981; Cifuentes et al., 1982; Andebrhan, 1985; Andebrhan et al., 1993), a vassoura-de-bruxa está apenas começando a ser estudada em cupuaçuzeiro, visto tratar-se de uma cultura antes basicamente explorada de forma extrativa, e agora, em plena expansão de áreas cultivadas racionalmente. Há registros sobre o comportamento da vassoura-de-bruxa no cupuaçuzeiro e a influência de variáveis climáticas (Nunes et al., 1996; Stein et al., 1996, 1997), porém, sem comprovações estatísticas da influência dessas variáveis sobre a doença.

O objetivo deste trabalho foi acompanhar o desenvolvimento de vassouras-de-bruxa vegetativas provocadas por C. perniciosa em cupuaçuzeiro, desde a sua emissão até a produção de basidiocarpos.

\section{Material e Métodos}

O experimento foi conduzido no período de junho/91 a maio/94, no campo experimental de fruteiras da Embrapa-Centro de Pesquisa Agropecuária do Trópico Úmido, em Belém, PA $\left(1^{\circ} 28^{\prime} \mathrm{S} ; 48^{\circ} 27^{\prime} \mathrm{W}\right)$, em plantas de cupuaçuzeiro de pé franco com sete anos de idade. O plantio foi feito em triângulo equilátero, com lados de $10 \mathrm{~m}$, em área de sub-bosque destocada, sombreada parcialmente por leguminosas arbóreas.

Durante a execução do experimento, os tratos culturais realizados no plantio seguiram as recomendações do sistema de produção do cupuaçuzeiro (Calzavara et al., 1984). Nas linhas de plantio, o coroamento foi feito a cada três meses. Nas entrelinhas, foi feita a roçagem mecanizada, a cada quatro a seis meses. Com vistas ao fornecimento do inóculo do patógeno, a área experimental foi selecionada em local próximo a um plantio cinqüentenário de cupuaçuzeiro, infestado pela vassoura-de-bruxa. As plantas marcadas foram podadas uma única vez, no início do experimento.

Para a execução do experimento, cinco plantas foram marcadas, na primeira semana de cada mês registrandose: número de vassouras vegetativas emitidas, duração dos períodos verde e de secamento, período pré-frutificativo, e produção de basidiocarpos. O delineamento experimental foi inteiramente casualizado, com 12 tratamentos, correspondentes aos meses do ano, e cinco repetições, correspondentes às plantas acompanhadas por mês.

Os dados de clima foram obtidos da estação climatológica da Embrapa, situada a $200 \mathrm{~m}$ da área experimental. As variáveis climáticas (umidade relativa do ar máxima, brilho solar e precipitação pluvial) foram correlacionadas com as variáveis epidemiológicas (emissão de vassouras, período verde, período de secamento, período pré-frutificativo e produção de basidiocarpos), utilizando técnica paramétrica (Pearson) ou nãoparamétrica (Spearman). As análises foram feitas pelo programa estatístico STATISTICA (Statistica..., 1995).

\section{Resultados e Discussão}

A emissão de vassouras vegetativas na área estudada foi observada em todos os meses do ano, mas foi mais concentrada nos meses de julho e agosto (Tabela 1). No cupuaçuzeiro, a presença de lançamentos jovens é registrada durante a maior parte do ano (Calzavara et al., 1984; Venturieri, 1993), favorecendo, assim, a ocorrência da vassoura-de-bruxa, uma vez que a flutuação ocorrida na quantidade de vassouras emitidas está relacionada com a presença de inóculo do patógeno no ambiente, e de tecidos receptivos na planta, além de fatores climáticos (Cifuentes et al., 1982).

A correlação entre a emissão de vassouras vegetativas e as variáveis climáticas estudadas foi não-significativa, apresentando tendência negativa em relação à umidade relativa do ar máxima e precipitação pluvial, e positiva, com relação ao brilho solar (Tabela 2). Isso indica que os fatores climáticos avaliados não interferiram diretamente na emissão de vassouras.

Os períodos mínimo e máximo em que as vassouras permaneceram verdes foram de 34,9 e 65,2 dias, respectivamente (Tabela 1). Na cultura do cacau, o período verde variou entre 19,6 e 49 dias (Hernandez, 1981). Correlação positiva com brilho solar e negativa com umidade relativa do ar máxima e precipitação pluvial, ambas não-significativas, foram detectadas quanto a essa variável (Tabela 2).

As vassouras verdes secaram em período variável entre 5,8 e 10 dias, da base para o ápice (Tabe- 
la 1), sendo a umidade relativa do ar máxima e a precipitação pluvial positivamente correlacionadas, de forma não-significativa, ao período de secamento das vassouras (Tabela 2).

Após sua emissão, as vassouras demoraram de 79,5 a 347 dias para iniciar a produção de basidiocarpos (Tabela 1). Em cacau, o período pré-frutificativo varia de 126 a 546 dias (Hernandez, 1981), estando sua duração relacionada com as condições ambientais, principalmente se há ausência de chuvas por mais de dois meses (Baker et al., 1941). A correlação desse parâmetro com a umidade relativa do ar máxima e com a precipitação pluvial foi negativa, porém, não-significativa (Tabela 2 ).

A produção de basidiocarpos foi detectada e computada a partir de maio/1992 até abril de 1994, com picos em junho (Figura 1). Estudos anteriores sobre a epidemiologia da vassoura-de-bruxa do cupuaçuzeiro conduzidos no campo experimental da Embrapa apontam a precipitação pluvial como a variável climática que mais influenciou na produção de basidiocarpos de C. perniciosa (Nunes et al., 1996). Estudos em cacau mostram a importância da umidade atmosférica (precipitação, neblina, orvalho e umidade relativa) na produção de basidiocarpos, na liberação e dispersão de basidiósporos (Andebrhan, 1988), na infecção, e na sincronização desses eventos (Purdy \& Schmidt, 1996). No presente trabalho, as análises de correlação entre as variáveis epidemiológicas estudadas e as variáveis climáticas indicam que a precipitação pluvial foi não-significa-

Tabela 2. Coeficientes de (r) entre variáveis climáticas e epidemiológicas relacionadas a vassouras-de-bruxa vegetativas provocadas por Crinipellis perniciosa em cupuaçuzeiro (Theobroma grandiflorum), em área experimental da Embrapa-CPATU, em Belém, PA, no período de junho/91 a maio/94.

\begin{tabular}{lccc}
\hline Variável & \multicolumn{3}{c}{ Variável climática } \\
\cline { 2 - 4 } epidemiológica & $\begin{array}{c}\text { Umidade } \\
\text { relativa do } \\
\text { ar máxima }\end{array}$ & $\begin{array}{c}\text { Brilho } \\
\text { solar }\end{array}$ & $\begin{array}{c}\text { Precipitação } \\
\text { pluvial }\end{array}$ \\
\hline Emissão de vassouras & $-0,56^{\mathrm{ns}}$ & $0,55^{\mathrm{ns}}$ & $-0,46^{\mathrm{ns}}$ \\
Período verde & $-0,26^{\mathrm{ns}}$ & $0,44^{\mathrm{ns}}$ & $-0,33^{\mathrm{ns}}$ \\
Período de secamento & $0,44^{\mathrm{ns}}$ & $-0,52^{\mathrm{ns}}$ & $0,49^{\mathrm{ns}}$ \\
Período pré-frutificativo & $-0,16^{\mathrm{ns}}$ & $0,28^{\mathrm{ns}}$ & $-0,22^{\mathrm{ns}}$ \\
\hline
\end{tabular}


tiva na produção de basidiocarpos $\left(r=-0,33^{\mathrm{ns}}\right)$. Porém, a umidade relativa do ar máxima e o brilho solar, com correlações negativa e positiva, respectivamente, foram significativas (Figura 1). Isso pode explicar a ausência de basidiocarpos nos períodos mais chuvosos do ano, quando o número de horas de brilho solar é bem menor e a umidade relativa do ar máxima é mais alta.

O conhecimento do comportamento das vassouras-de-bruxa vegetativas ao longo do ano é fundamental para o embasamento de medidas de controle da doença no campo, uma vez que a interrupção do ciclo da doença pode ser feita mediante a poda dessas vassouras durante o período pré-frutificativo, evitando, assim, a produção de basidiocarpos, principal fonte de propagação da vassoura-de-bruxa (Baker et al., 1941; Andebrhan et al., 1993).
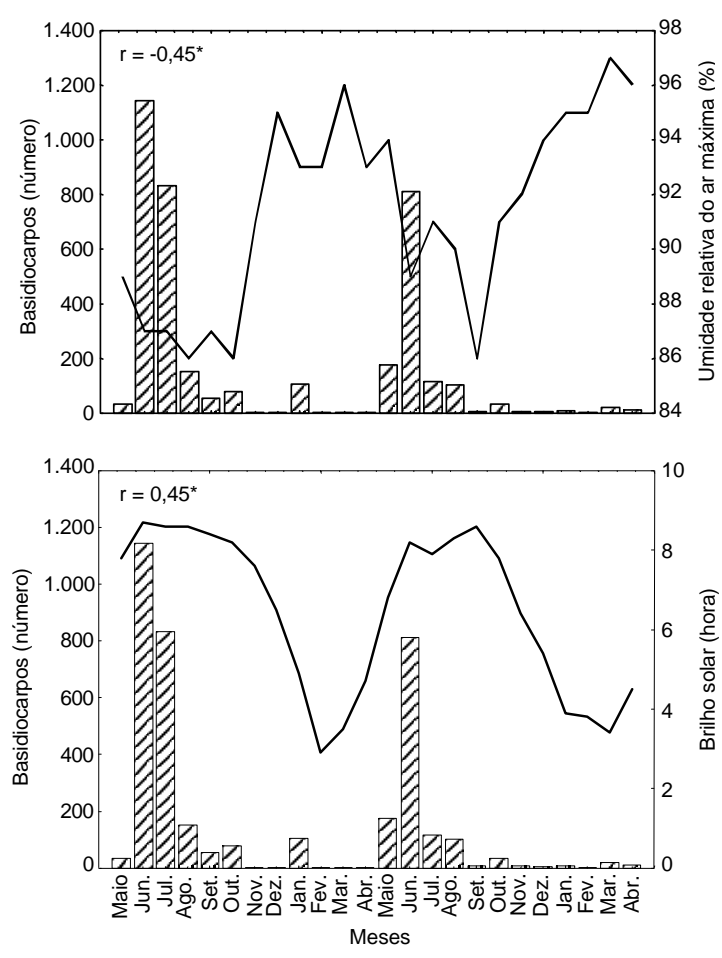

Figura 1. Relação entre o número de basidiocarpos ( $\square$ ) de C.perniciosa em vassouras vegetativas de cupuaçuzeiro, em relação à umidade relativa do ar máxima e ao brilho solar, no período de maio/92 a abril/94.

\section{Conclusões}

1. As vassouras vegetativas no cupuaçuzeiro ocorrem durante todo o ano, com picos nos meses de julho e agosto.

2. Após sua emissão, as vassouras vegetativas permanecem verdes por $49,9 \pm 19,4$ dias ( \pm d.p.) e se-

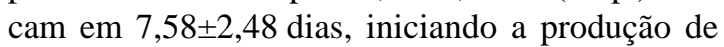
basidiocarpos após 212,2 $\pm 87,1$ dias.

3. A produção de basidiocarpos ocorre mais freqüentemente a partir de maio, com pico em junho.

4. A umidade relativa do ar máxima e o brilho solar influem de forma significativa na produção de basidiocarpos, e correlacionam negativa e positivamente, respectivamente, com essa variável.

\section{Agradecimentos}

A Moacyr B. Dias-Filho, pela análise estatística dos dados e pela revisão da primeira versão do trabalho.

\section{Referências}

ALVES, R. M.; CORREA, J. R. V.; GOMES, M. R. O; FERNANDES, G. L. C. Melhoramento genético do cupuaçuzeiro (Theobroma grandiflorum) no Estado do Pará. In: SEMINÁRIO INTERNACIONAL SOBRE PIMENTA-DO-REINO E CUPUAÇU, 1., 1996, Belém. Anais... Belém : Embrapa-CPATU/JICA, 1997. p. 127-146. (Embrapa-CPATU. Documentos, 89).

ANDEBRHAN, T. Rain water as a factor in the dissemination of basidiospores of Crinipellis perniciosa (Stahel) Singer within cacao trees. In: INTERNATIONAL COCOA RESEARCH CONFERENCE, 10., Santo Domingo, 1987. Proceedings... Lagos : Cocoa Producers' Alliance, 1988. p. 363-366.

ANDEBRHAN, T. Studies on the epidemiology and control of witches' broom disease of cacao in the Brazilian Amazon. In: INTERNATIONAL COCOA RESEARCH CONFERENCE, 9., Lome, 1984. Proceedings... Lagos : Cocoa Producers' Alliance, 1985. p. 395-402.

ANDEBRHAN, T.; COSTA, J. C. B; CARVALHO, A. L. P.; ALBUQUERQUE, P. Comparative epidemiology experiment: Brazil. In: RUDGARD, A. C.; MADDISON, A. C.; ANDEBRHAN, T. (Ed.). Disease management in 
cocoa: comparative epidemiology of witches' broom. London : Chapman \& Hall, 1993. p. 33-92.

BAKER, R.; CROWNDY, S. H.; THOROLD, C. A. Witches' broom disease investigations: seasonal variations in the intensity of infections and their effect on control measures. Tropical Agriculture, St. Augustine, v. 18, p. 107-116, 1941.

CAlZAVARA, B. B. G.; MULleR, C. H.; KAWAGE, O. N. C. Fruticultura tropical: o cupuaçuzeiro: cultivo, beneficiamento e utilização do fruto. Belém : EmbrapaCPATU, 1984. 101 p. (Embrapa-CPATU. Documentos, 32).

CIFUENTES, C.; MAYORGA, M.; PRIETO, E.; RONDON, G.; TOVAR, G. Infection and sporulation by Crinipellis perniciosa (Stahel) Singer, causal agent of witches' broom disease of cacao in Guamal, Colombia. In: INTERNATIONAL COCOA RESEARCH CONFERENCE, 8., Cartagena, 1981. Proceedings... Lagos : Cocoa Producers' Alliance, 1982. p. 485-492.

HERNANDEZ, F. A. Algunos aspectos de la biología del hongo Crinipellis perniciosa (Stahel) Singer en la región de Uraba, Colombia. Santa Fe de Bogota : Universidad Nacional de Colombia, 1981. 82 p. Tesis Magíster Scientiae.

NUNES, A. M. L.; ALBUQUERQUE, F. C.; OLIVEIRA, R. P.; SÁ, T. D. A.; NUNES, M. A. L.; SHIMIZU, O. Epidemiologia da vassoura-de-bruxa do cupuaçuzeiro. In: EMBRAPA. Centro de Pesquisa Agroflorestal da Amazô- nia Oriental (Belém, PA). Geração de tecnologia agroindustrial para o desenvolvimento do trópico úmido. Belém : Embrapa-CPATU/JICA, 1996. p. 83-105. (Embrapa-CPATU. Documentos, 85).

PURDY, L. H.; SCHMIDT, R. A. Status of cacao witches' broom: biology, epidemiology and management. Annual Review of Phytopathology, Palo Alto, v. 34, p. 573-594, 1996.

STATISTICA for Windows: general conventions and statistics. Tulsa : StatSoft, 1995. v. 1.

STEIN, R. L. B.; ALBUQUERQUE, F. C.; NASCIMENTO, R. M. Vassoura-de-bruxa do cupuaçuzeiro: observações de campo. In: SEMINÁRIO INTERNACIONAL SOBRE PIMENTA-DO-REINO E CUPUAÇU, 1., 1996. Belém. Anais... Belém : Embrapa-CPATU/JICA, 1997. p. 333-339. (Embrapa-CPATU. Documentos, 89).

STEIN, R. L. B.; ALBUQUERQUE, F. C.; ROCHA NETO, O. G.; CONCEIÇÃO, H. E. O.; BASTOS, C. N.; ENDO, T.; ITO, T. Biologia e fisiologia de Crinipellis perniciosa do cupuaçuzeiro. In: EMBRAPA. Centro de Pesquisa Agroflorestal da Amazônia Oriental (Belém, PA). Geração de tecnologia agroindustrial para o desenvolvimento do trópico úmido. Belém : Embrapa-CPATU/ JICA, 1996. p. 59-82. (Embrapa-CPATU. Documentos, 85).

VENTURIERI, G. A. Cupuaçu: a espécie, sua cultura, usos e processamento. Belém : Clube do Cupu, 1993. 108 p. 